\title{
ANÁLISE DA ADEQUAÇÃO DA TEORIA DO RELACIONAMENTO INTERPESSOAL EM GRUPOS CONDUZIDOS POR ENFERMEIRA ${ }^{1}$
}

\author{
Analysis of the Adequacy of Interpersonal Relations Theory in Nurse-Led Groups ${ }^{1}$ \\ Análisis de la Adecuación de la Teoría del Relacionamiento Interpersonal en Grupos \\ Conducidos por Enfermera ${ }^{1}$
}

Maria Adelane Alves Monteiro²

Lorita Marlena Freitag Pagliuca ${ }^{3}$

\section{RESUMO}

A Teoria do Relacionamento Interpessoal em Enfermagem (Peplau, 1993) descreve relações entre a enfermagem e o paciente. Neste estudo objetivou-se analisar a adequação desta teoria para aplicação em grupos conduzidos por enfermeira. Estudo analítico apoiado em modelo de análise de teorias de enfermagem. A metodologia constou da seleção de artigos disponíveis no MEDLINE com delimitação de tempo de 1996 a 2006, usando os descritores psicoterapia de grupo e teoria de enfermagem e a palavra-chave grupos. Apenas um artigo a plicou a teoria em discussão, e sua análise demonstrou que a relação enfermeira-cliente e as fases e papéis da Teoria do Relacionamento Interpessoal em Enfermagem são adequados a intervenções grupais.

Palavras-chave: Teoria de Enfermagem. Psicoterapia de Grupo. Enfermagem.

\begin{abstract}
The Theory of Interpersonal Relations in Nursing (Peplau, 1993) describes relations between nursing and patients. This study aimed to analyze the adequacy of this theory for application in groups led by a nurse. We carried out an analytic study, based on model analysis of theories of Nursing. The research methodology included the selection of articles available in MEDLINE which were published between 1996 and 2006, using the descriptors psychotherapy group and nursing theory as well as the keyword. Only one article applied the theory under study, and its analysis demonstrated that the nurse-client relation and the phases and roles of the Theory of Interpersonal Relations in Nursing are adequate for group interventions.
\end{abstract}

Keywords: Nursing Theory. Psychotherapy Group. Nursing.

\section{Resumen}

La Teoría del Relacionamiento Interpersonal en Enfermería (Peplau, 1993) describe relaciones entre la enfermería y el paciente. La finalidad de este estudio fue analizar la adecuación de esta teoría para aplicación en grupos conducidos por enfermera. Estudio analítico apoyado en el modelo de análisis de teorías de Enfermería. La metodología consistió en la selección de artículos disponibles en MEDLINE con delimitación de tiempo de 1996 a 2006 y usando los descriptores psicoterapia de grupo y teoría de enfermería, además de la palabra clave grupos. Sólo un artículo aplicó la teoría en discusión y su análisis demostró que la relación enfermeracliente y las fases y papeles de la Teoría del Relacionamiento Interpersonal en Enfermería son adecuados a intervenciones grupas.

Palabras clave: Teoría de Enfermería. Psicoterapia de Grupo. Enfermería. 


\section{INTRODUÇÃO}

Por necessidade, o homem é um ser de natureza gregária, antes mesmo de ter consciência deste fato. Como é notório, grande parte das atividades cotidianas do ser humano é desenvolvida em grupo. Tal constatação denota a exigência de estudos sobre o tema ${ }^{1}$.

No dia-a-dia do enfermeiro, o trabalho grupal é uma realidade vivenciada em todo o período da sua formação acadêmica e traz benefícios como estratégia para a atividade profissional de enfermagem ${ }^{2}$. Conforme evidenciado, o grupo é um instrumento de grande valia para o enfermeiro no planejamento de sua intervenção, sobre tudo por propiciar caminhos para o cuidado emocional ${ }^{3}$. Neste estudo, grupo é entendido como uma entidade em si, com qualidades que podem diferir daquelas de cada membro em particular. Isoladamente não é possível analisar um grupo, especialmente quando se trata de utilizá-lo como método de ajuda entre pessoas?.

Ao se desenvolver a dissertação de mestrado na qual se investigou, por meio do trabalho em grupo, a vivência de puérperas que acompanhavam o filho recém-nascido hospitalizado, foi possível verificar que a literatura dispõe de trabalhos sobre a experiência da utilização da estratégia de grupo nas mais diversas situações ${ }^{4}$. Entretanto, poucos são os estudos que testam a aplicação de teorias na abordagem grupal elaborados por enfermeiros ${ }^{5-10}$ de forma a contribuir para a construção de estratégias de cuidado eficazes. Segundo se percebeu, a fundamentação dos estudos existentes sobre processo grupal advém da psicoterapia.

Uma teoria deve ser capaz de direcionar a prática, caracterizando e apontando os componentes essenciais que darão identidade aos fenômenos da profissão ${ }^{11}$. De acordo com o entendimento da autora, as teorias devem guiar a prática, assim como o conhecimento que dá origem às teo rias nasce dessa prática. Logo, conhecimento, prática e teoria estão inter-relacionados. No entanto, antes de ser aplicada na realidade profissional, uma teoria deve ser estudada. Deste modo, se evitará aceitá-la sem nenhuma reflexão, discussão ou exame. Contudo, o julgamento não deve ser analisado sob o ponto de vista negativo, pois a crítica é o primeiro passo para se melhorar uma teoria ${ }^{11}$.

Diante destas exigências, escolheu-se para este estudo 0 seguinte objetivo: analisar a adequação da Teoria do Relacionamento Interpessoal em Enfermagem de Peplau, a partir da sua aplicação em grupos conduzidos por enfermeira.

\section{REFERENCIAL TEÓRICO-METODOLÓGICO}

0 presente estudo teve como referencial teóricometodológico um método de análise de teorias, o qual consiste num processo que envolve a classificação e definição da estrutura da teoria e um julgamento posterior. Questiona-se quanto ao ambiente onde a teoria ocorre, sobre seus elementos e o que se espera alcançar com o seu uso, compreendendo sua estrutura (conteúdo, contexto, processo e metas). Este é um exercício a ser realizado por todo enfermeiro antes de aplicar determinada teoria para identificar seus objetivos e se ela direciona a prática de enfermagem. Reconhecer o nivel de desenvolvimento da teoria, classificandoa como descritiva ou explanatória; investigá-la segundo seu princípio (essência), interpretação (visão de mundo do autor, cenário onde a teoria se insere - existencial ou essencial) e método (dialético, logístico, problematizador ou operacional) são passos fundamentais para a escolha da teoria adequada a cada situaçãa $0^{11}$.

Analisada e classificada a teoria, torna-se necessário julgála. Esse julgamento pode partir de duas posições: simplesmente questão de gosto pessoal ou mediante utilização de critérios distintos que constituem a crítica interna ou externa. A crítica interna lida com os componentes de clareza, consistência, adequação, desenvolvimento lógico e nível de desenvolvimento da teoria. A externa trata dos elementos de convergência, utilidade, importância, discriminação, extensão e complexidade da teoria. Optou-se nesse estudo pelo critério de adequação, ou seja, quando uma teoria consegue contemplar 0 assunto com que se pretende trabalhar, seja este amplo ou limitado. As prescrições de uma teoria adequada devem cobrir a extensão estipulada pelo autor. Conseqüentemente este critério pode refletir tanto a crítica interna quanto a externa ${ }^{11}$.

A Teoria do Relacionamento Interpessoal em Enfermagem foi elaborada a partir da experiência teórica de trabalho com pacientes hospitalizados e com distúrbios de relacionamento, na qual sua intervenção se volta para a relação enfermeirapaciente, uma relação humana entre uma pessoa que está necessitada de cuidados de saúde e uma enfermeira com formação especializada em reconhecer e responder às necessidades de ajuda ${ }^{12}$.

Nesta teoria, enfermagem é definida como um processo interpessoal e terapêutico, consistindo em ações que exigem a participação entre duas ou mais pessoas que se beneficiam desta interação. Saúde é descrita como símbolo lingüístico, movimento progressivo da personalidade na busca de uma vida construtiva, produtiva, pessoal e comunitária. A relação interpessoal é aquela em que duas pessoas passam a se conhecer suficientemente bem de forma que seja possível enfrentar os problemas que surgem de forma cooperativa ${ }^{12}$. Não há referência direta à sociedade ou ambiente, mas a é enfermeira estimulada a considerar a cultura e os costumes das pessoas envolvidas no processo de interaçãa ${ }^{13}$.

De acordo com a literatura, o processo de relacionamento interpessoal da enfermeira com seu paciente se desenvolve em quatro fases seqüenciais claramente perceptíveis, com momentos sobrepostos ou inter-relacionados, quais sejam:

(1) orientação - momento em que enfermeira e paciente têm o primeiro contato, quando ambos identificam e compreendem as necessidades afetadas do paciente; (2) identificação - fase em que o indivíduo começa a experimentar a sensação de ser 
capaz de lidar com o problema que o aflige. Essa transformação diminui os sentimentos de desamparo e cria motivação para 0 enfrentamento da sua necessidade; (3) exploração - neste momento o paciente começa a perceber vantagens do encontro terapêutico, isto é, ele começa a controlar o problema, e recebe ajuda. Como parte de suas atribuições, cabe à enfermeira utilizar instrumentos de comunicação, como o esclarecimento, a escuta, a orientação, a interpretação, entre outros, para oferecer recursos favoráveis à adaptação do paciente; (4) resolução - momento em que as necessidades do paciente já foram supridas pelos esforços conjuntos. Nessa fase, enfermeira e paciente devem desfazer os laços e pôr fim ao relacionamento terapêutico. Quando a relação é bem sucedida, o paciente afasta-se da enfermeira, e torna-se independente, forte e amadurecido ${ }^{12}$.

Cada uma destas fases se caracteriza por papéis ou funções desempenhadas pela enfermeira, à medida que ela e o paciente aprendem a trabalhar conjuntamente para resolver dificuldades. Entre estes papéis mencionavam-se os seguintes: 0 de estranho, 0 de recurso, 0 de professor, 0 de substituto, 0 de conselheiro e o de líder.

0 papel de estranho é desempenhado durante 0 contato inicial (fase de orientação) e implica aceitação do paciente pela enfermeira e vive-versa. Neste deve haver respeito e interesse positivo. 0 de recurso é quando se oferecem informações específicas para compreensão de um problema ou situações novas e a enfermeira proporciona respostas específicas às perguntas habitualmente formuladas em relação a um problema maior. 0 de professoré quando se compar tilham conhecimentos sobre uma necessidade ou interesse. Neste caso, o ensino avança sempre a partir do que o paciente já conhece e se desenvolve em torno do seu interesse. No papel de substituto, o paciente considera inconscientemente a enfermeira como outra pessoa, a qual pode simbolizar uma figura materna ou substituir o papel de uma irmã do paciente, por exemplo. 0 papel de conselheiro é desempenhado quando se auxilia outra pessoa no reconhecimento, enfrentamento, aceitação e solução de problemas; e o de líder é desenvolvido por meio da capacidade da enfermeira de sentar-se junto ao paciente, observar, compreender como uma situação pode afetá-10, analisar o que sucede no íntimo do paciente e apreciar 0 desenvolvimento da relação interpessoal entre ambos ${ }^{12}$.

A teoria de Peplau não se limita à seleção enfermagempaciente, pois uma perspectiva mais ampla estendeu a relação terapêutica aos membros familiares, outros profissionais e pessoas com problemas semelhantes ${ }^{14}$. Conforme se entende, as relações entre pessoas com problemas semelhantes podem se desenvolver via grupos e nesse tipo de relacionamento podem ocorrer apoio mútuo, compartilhamento, validação e desenvolvimento de papéis positivos. No entanto, a experiência apresentou esta abordagem em relação a um paciente individualmente, ressaltando apenas a possibilidade deste tipo de relação, e não a aplicação da teoria em grupos.

\section{PERCURSO METODOLÓGICO}

Trata-se de um estudo analítico, com coleta de dados bibliográficos realizada em novembro de 2006. A investigação foi efetuada via Internet, junto à BVS/BIREME (Biblioteca Virtual em Saúde do Centro Latino-Americano e do Caribe de Informação em Ciências da Saúde), onde foram pesquisados os artigos de periódicos de 1996 a 2006, indexados na base de dados MEDLINE (Literatura Internacional em Ciências da Saúde). A lista dos Descritores em Ciências da Saúdes (DeCS), edição 2006, foi consultada e apresentava como descritor aproximado para grupo prática de grupo e psicoterapia de grupo. Como observado, o primeiro é definido nos DeCS como uma forma de organização de trabalho da classe médica, 0 que fundamentou a escolha do segundo, que diz respeito a uma forma de terapia da qual dois ou mais pacientes participam sob a orientação de um ou mais psicoterapeutas com o propósito de resolver problemas emocionais, sociais ou psíquicos. Optou-se também pela palavra-chave grupos. Quanto à intenção de descobrir referenciais teóricos embasando a abordagem grupal, decidiu-se por teoria de enfermagem como descritor.

Na busca inicial utilizou-se como primeiro descritor psicoterapia de grupo. Sob esta denominação, encontraramse 1.880 artigos. Ao se proceder à seleção com o segundo descritor, teoria de enfermagem, somente cinco trabalhos foram identificados. Outra tentativa empregou como primeiro descritor teoria de enfermagem. Neste caso localizaram-se 2.142 publicações. No refinamento com a palavra-chave grupos, identificaram-se 47 artigos.

Os dois grupos foram somados com a posterior leitura detalhada dos resumos dos 52 artigos obtidos. Detectou-se que apenas 10 destes relatavam a experiência da abordagem grupal a partir da testagem de teorias, entre os quais um estudo ilustrava a aplicação da teoria de Peplau em grupos de psicoterapia9.

\section{APLICAÇÃO DA TEORIA DO RELACIONAMENTO INTERPESSOAL DE ENFERMAGEM EM GRUPOS DE PSICOTERAPIA}

\section{Fases da relação enfermeira-paciente em grupos de psicoterapia}

Conforme o estudo em análise afirma, as fases de desenvolvimento de um grupo podem ocorrer paralelamente ao desenvolvimento da relação do paciente individualmente com a enfermeira. De modo geral, a fase de orientação acontece antes de o paciente entrar no grupo. Nesse primeiro contato, 0 paciente avalia a enfermeira como pessoa e como psicoterapeuta, o que corresponde a uma parcela dos fatores que influenciam sua decisão de participar ou não do grupo. Se considerar a enfermeira uma pessoa confiável, o paciente decide se tornar um membro do grupo. Da mesma forma, a enfermeira decide se a terapia de grupo é apropriada para 0 paciente, e se este seria adequado ao grupo. Todas essas decisões são possíveis pelo padrão de relacionamento entre 0 paciente e a enfermeira ${ }^{9}$. 
Uma vez estabelecidos estes parâmetros, enfermeira e paciente identificam e compreendem as dificuldades e necessidades afetadas do paciente. Nessa fase, é essencial a enfermeira trabalhar em cooperação com o paciente, no sentido de que, juntos, reconheçam, esclareçam e definam o problema existente ${ }^{13}$. No artigo analisado, porém, não há referência de que nessa fase seja possível a identificação dos problemas de relacionamento do paciente em conjunto, ou seja, enfermeira e paciente chegam a um consenso, mesmo se esse processo ocorrer antes da participação do paciente no grupo.

No grupo, os participantes do processo interpessoal são 0 paciente, a enfermeira e todos os outros membros. Para exemplificar a fase de identificação, a autora assim se pronuncia: é possível que um membro do grupo se perceba identificando seu problema ou limitação. Do mesmo modo, os outros membros do grupo começam a identificá-lo a partir da sua característica mais expressiva, o que normalmente corresponde ao seu problema. Em seguida, por meio do discurso diário desse membro, todos os participantes identificam seu padrão de relacionamento e sua necessidade interpessoal para manter suas relações saudáveis?.

A teoria se refere à fase de identificação da seguinte forma: nesse momento se declara a primeira impressão do paciente e este acredita perceber quem pode oferecer-Ihe algo, e então seleciona aqueles capazes de ajudá-lo, demonstrando segurança. Os pacientes se reconhecem facilmente nas pessoas que os aceitam e, deste modo, se identificam totalmente com as enfermeiras. Além disso, conforme esperam, estas atenderão seus desejos sem exigir nada em troca. Ao se identificarem com as enfermeiras, os pacientes ofazem com base nos serviços a eles prestados e que consideram úteis ${ }^{12}$. Portanto, deve haver uma identificação positiva do paciente com a enfermeira e dos membros do grupo com esta e entre si. Contudo, isto não é mencionado no artigo.

$\mathrm{Na}$ fase seguinte, o paciente explora todos os diversos bens e serviços existentes à sua dis posição, com vistas a seus interesses e necessidades. Com esta finalidade, faz pleno uso dos serviços então oferecidos. Para ele, porém, os problemas de enfermagem mais complexos derivam da exploração da situação, e não da base de identificação positiva com as enfermeiras ou da liberdade de fazer uso dos serviços prestados ${ }^{12}$.

Consoante a teoria, e segundo 0 artigo discorre, nessa fase 0 paciente especula o conhecimento e habilidade da enfermeira para mudar relações problemáticas. 0 como e 0 porquê do problema devem ser explorados. Igualmente, 0 membro do grupo deve ser questionado sobre como ele chegou a esse padrão de comportamento. Para isso, é fundamental a enfermeira manter uma atmosfera desinibidora da comunicação, ajudando o paciente na exploração de todos os caminhos que 0 levam ao bem-estar. Conforme a autora evidencia, na fase de exploração o uso dos passos do processo de aprendizagem no grupo pode ajudar o membro a descrever seu passado, observar seus efeitos no presente, analisar suas relações, formular um modo de mudança, validar suas formulações e buscar a validação dos outros membros?.
De acordo com o exemplo anterior, a autora ressalta que 0 membro do grupo na fase de resolução começa a demonstrar que ele poderia testar, integrar e utilizar o conhecimento ali adquirido. Ele passa a assumir a ansiedade em controlar-se para não continuar cometendo os mesmos erros e já é capaz de perceber quando assume determinados padrões. Nessa fase, o membro do grupo já começa a tentar resolver seu problema?.

Como observado, a autora do estudo em análise não refere que essa última fase do processo interpessoal proposto na Teoria de Relacionamento Interpessoal em Enfermagem ${ }^{12}$ é caracterizada pelo momento em que as necessidades do paciente já foram supridas pelos esforços conjuntos e ele se sente capaz de cuidar-se. Assim, a fase de resolução implica a liberação gradual das pessoas que the proporcionaram ajuda e a criação e fortalecimento da capacidade de atuar por si mesmo. Ainda como observado, o afastamento do paciente da enfermeira, ao se tornar independente, assim como a forma como ele ocorre ou deve ocorrer no grupo não são mencionados no artigo.

\section{Papéis da enfermeira em grupos de psicoterapia}

Segundo mencionado, durante a evolução do processo de relacionamento interpessoal no grupo, a enfermeira assume diferentes papéis, tais como:

\section{0 de estranho}

Os pacientes que participarão de um grupo, antes de iniciarem esta atividade, fazem terapia individual, embora sejam apenas algumas sessões. Nas primeiras sessões, enfermeira e paciente são estranhos um ao outro. Durante este período, é importante a enfermeira respeitar a necessidade de distância do paciente. Quando este entra no grupo, as informações sobre a enfermeira e sobre ele próprio vão aumentand $0^{9}$. Em virtude deste fato, em um mesmo grupo pode haver pacientes e enfermeira estranhos um ao outro e membros que conhecem a enfermeira por mais tempo. Desta forma, a entrada de um novo paciente no grupo constitui para o líder um grande aprendizado de negociação tanto sobre 0 conteúdo da vida do paciente quanto em relação aos padrões de relacionamento interpessoais dos participantes.

Nessa perspectiva, ao se tratar de grupos abertos, nos quais a entrada e a saída dos membros são eventos freqüentes, o papel de estranho também é desempenhado pelo novo membro ao entrar no grupo em relação aos outros membros, mas estes também se constituem estranhos ao recém-chegado.

\section{$\mathbf{O}$ de recurso}

No papel de pessoa de recurso, a enfermeira "proporciona respostas específicas às perguntas normalmente formuladas com relação a um problema maior"12:47. Esse papel é constantemente desempenhado pela enfermeira que conduz grupos de psicoterapia. Por exemplo, quando um membro do grupo expõe algo com o qual os outros membros não concordam e estes não possuem argumentos para convencê-lo quanto a uma atitude ou compor tamento não saudável, a enfermeira é solicitada a opinar sobre o caso, como forma de recurso para que o paciente possa mudar seu pensamento ${ }^{9}$. 


\section{0 de professor}

De modo geral, o papel de professor de um terapeuta de grupo envolve assuntos interpessoais. A melhor maneira de ensinar comportamento interpessoal é pelo exemplo da enfermeira, da mesma forma como é experienciado com os pais. Um dos ensinamentos da enfermeira aos pacientes em terapia de grupo é a tolerância a um novo comportamento, tendo em vista a diversidade de personalidades e experiências vivenciadas pelos membros de um mesmo grupo ${ }^{9}$.

\section{$\mathbf{O}$ de substituto}

Freqüentemente os pacientes atribuem às enfermeiras papéis de substitutos. 0 paciente associa a relação mantida com a enfermeira com a de alguém com quem já tenha se relacionado, seja um membro familiar ou uma figura conhecida representativa (professor, outra enfermeira, etc.) ${ }^{12}$. Inevitavelmente a enfermeira deve ser vista como uma mãe ou o grupo como uma família substituta do paciente. Por meio do processo de transferência, este associa a enfermeira e os membros do grupo mais próximos a pessoas do seu contato, considerando 0 apoio recebido ${ }^{9}$. Esse fenômeno é muito comum em grupos.

\section{$\mathbf{O}$ de conselheiro}

Esse papel é desempenhado quando a enfermeira auxilia outra pessoa no reconhecimento, enfrentamento, aceitação e solução dos problemas.

No papel de conselheiro, o terapeuta de grupo exercita a psicoterapia interpessoal propriamente dita. Juntamente com os membros do grupo, a enfermeira examina os problemas de relacionamento interpessoal destes, guiando-os pelos passos do processo de aprendizagem, durante as fases de relacionamento. Ela deve evitar fazer terapia individual. Mas os membros do grupo devem conduzir os trabalhos sob a direção sutil de líder, ajudando um ao outro a reconhecer e solucionar problemas interpessoais 9 .

Conforme se percebe, esse papel ocorre com maior freqüência na fase de exploração do relacionamento interpessoal em enfermagem em relação aos grupos de psicoterapia, nos quais a contribuição do enfermeiro torna-se fundamental. Tal papel, entretanto, deve permear todo o processo, pois num mesmo grupo pode haver pacientes em fases diferentes da relação. Crê-se ainda que esse papel também possa ser assumido pelos membros do grupo, em especial por aqueles que já alcançaram um melhor nível de relacionamento interpessoal.

\section{0 de líder}

Ao analisar uma situação que pode estar afetando o paciente em seu relacionamento interpessoal, a partir da relação desenvolvida entre ambos, a enfermeira desempenha o papel de líder. Sua capacidade de compreender essa situação é o que sustenta esse papel.

0 papel do líder em um grupo é estimular os membros a expressar seus padrões interpessoais de relacionamento. Para a teorista, existe o líder democrático, e ela o descreve como aquele que permite ao paciente ser um participante ativo no planejamento de mudança ${ }^{13}$. Este modelo é bastante trabalhado em terapia de grupo quando a enfermeira permite aos pacientes conduzir o grupo, não determinando programação ou assuntos para serem discutidos pelos pacientes. Neste caso, a dinâmica do grupo acontece naturalmente, a enfermeira observa as interações e guia os pacientes para explorá-las 9 .

Em um grupo, as experiências vivenciadas são compartilhadas entre os participantes, e por meio delas é possivel identificar e analisar o padrão de relacionamento. Desta forma, pode-se inferir que o papel de líder é inerente a qualquer intervenção baseada na abordagem grupal, quando se executa o processo de estabelecimento e manutenção das metas do grupo, mediante interação, e compreende-se que, nesse caso, não necessariamente este deva ser desempenhado somente pela enfermeira coordenadora do grupo. A qualquer momento, pode haver a mudança de protagonista.

\section{ANÁLISE REFLEXIVA DA ADEQUAÇÃO DA TEORIA EM GRUPOS CONDUZIDOS POR ENFERMEIRA}

0 desenvolvimento deste estudo permitiu compreender que as fases do relacionamento interpessoal propostas na Teoria do Relacionamento Interpessoal de Enfermagem também ocorrem no processo grupal, embora muitas vezes não se possa prever o que irá acontecer em um grupo. Estas fases, assim como exposto pela teorista, também acontecem no grupo com momentos sobrepostos e inter-relacionados. Contudo, no caso de grupos com características de permanente e aberto, as fases de relacionamento podem não se desenvolver seqüencialmente.

Segundo se considera, a fase de orientação é aquela na qual as necessidades e os problemas de relacionamento do paciente são identificados. Ela se dá no contato inicial da enfermeira com 0 paciente e se entende até as primeiras sessões do grupo, pois, consoante se entende, 0 compartilhamento de experiências pode suceder durante todo o processo do grupo, e este é um fator que permite evidenciar as necessidades do paciente, tanto pela enfermeira quanto pelos outros membros.

No artigo em estudo, como observado, alguns aspectos da teoria não foram mencionados. Entretanto, conforme se acredita, eles podem perfeitamente acontecer no ambiente do grupo. Em qualquer tipo de relacionamento terapêutico, a finalização do encontro terapêutico, quando o paciente consegue resolver seu problema e torna-se independente, é o resultado esperado e desejável.

Como evidenciado, a relação enfer meira-paciente pode ser utilizada como base para desenvolver outros tipos de relações. Desta forma, é útil para a prática do enfermeiro, ao orientá-lo na condução do grupo e auxiliá-lo na compreensão do processo terapêutico que ocorre nesse ambiente. Ademais permite, pelo estabelecimento da relação saudável, o desenvolvimento de cuidados eficazes. Portanto, considera-se que a Teoria do Relacionamento Interpessoal em Enfermagem se adequa às intervenções grupais. 


\section{Referências}

1. Rogers CR. Grupos de encontro. 8aed. São Paulo (SP): Martins Fontes; 2002.

2.Câmara MFB, Damásio VF, Munari DB. Vivenciando os desafios do trabalho em grupo. Rev Eletr Enferm [on-line] 1999 out/dez; [citado 18 mar 2005]; 1(1): [aprox. 12 telas]. Disponível em: http:// www.fen.ufg.br/revista/revista1_1/09.htm

3. Munari DB, Ribeiro V, Lopes MM. Intervenção grupal com enfoque no cuidado emocional: relato de experiência. Rev Bras Enferm 2002 jul/ago; 55(4): 449-51.

4. Monteiro MAA. Abordagem grupal a puérperas com filhos recémnascidos hospitalizados. [ dissertação de mestrado]. Fortaleza(CE): Departamento de Enfermagem/UECE, 2005.

5. Arndt MJ, Horodynski MA. Theory of dependent-care in research with parents of toddlers: the NEAT project. Nurs Sci Q [on-line] 2004 out; [citado 15 nov 2006]; 17(4): [aprox.6 telas]. Disponível em: http://www.ncbi.nlm.nih.gov/pubmed/15359037

6. Kelley LS. Evaluating change in quality of life from the perspective of the person: advanced pratice of nursing and Parse's goal of nursing. Holist Nurs Pract [on-line] 1999 jul; [ citado 15 nov 2006]; 13(4): [aprox.12 telas]. Disponível em: http://www.hnpjournal.com/pt/ rehnpserLogin.htm;jsessionid= HpbXsnvhfX7 QMhkyhpCsHWIc7q

1 BrvYwNv]1 CQpM1 sgQS0Z64 VWy!327387095 !181195628!8091!-1

7. Strickland D. Applying Watson's Theory for caring among elders. J Gerontol Nurs [on-line] 1996 [citado 15 nov 2006]; 22 (7): [aprox.8 telas]. Disponível em: http://www.serach.ebscohost.com/ longin. aspx? direct= true $\& d b=c 8 h j i d=1 F D \&$ site $=$ ehost-live
8.Calladine ML. Nursing process for health promotion using King's theory. J Community Health Nurs [on-line] 1996; [citado 15 nov 2006]; 13(1): [aprox.8 telas]. Disponível em: http:// www.serach.epnet.com/login.aspx? direct= true $\alpha d b=c 8 h j i d=7 \mathrm{M} 8$

9. Lego S. The application of Peplau's Theory to group psychotherapy. J Psychiatr Ment Health Nurs [on- line] 1998 jun; [citado 15 nov 2006]; 5(3): [aprox.5 telas]. Disponível em: http://www. serach.epnet.com/login.aspx? direct $=$ true $\& d b=c 8 h j i d=81 \mathrm{~W}$

10. Cardoso TVM, Oliveira RMP, Loyola CMD. Um entendimento linear sobre a Teoria de Peplau e os princípios da reforma psiquiátrica brasileira. Esc Anna Nery Rev Enferm [on-line] . 2006 dez; [citado 10 abr 2008]; 10(4): [aprox. 6 telas]. Disponível em: <http:// w w w. portalbvsenf. e e r p.us p. br / scielo.php? script =sci_arttext $\alpha p i d=s 1414$ $81452006000400014 \&$ lng $=p t \& n r m=$ iso $>$. ISSN 1414-8145.

11. Barnum BS. Nursing theory: analysis, application, evaluation. 5 a ed. Philadelphia(USA): Lippincott Company; 1998.

12.Peplau H. Relaciones interpesonales em enfermería. Barcelona(ES): Masson-Salvat; 1993.

13. Pinheiro AKB. Depois do parto tudo muda: um novo olhar sobre adolescentes nutrizes. [tese de doutorado]. Fortaleza(CE): Departamento de Enfermagem /UECE; 2003.

14. Forchuk C, Jewell J, Schofield R, Sircelj M, Valledor T. From hospital to community: bridging therapeutic relationships. J Psychiatr Ment Health Nurs [on-line] 1998 ago; [citado 15 nov 2006]; (5): [aprox. 6 telas]. Disponível em: http://www.blackwell-synergy.com/servlet/ useragent? func $=$ showlssues $\&$ code $=$ jpm 